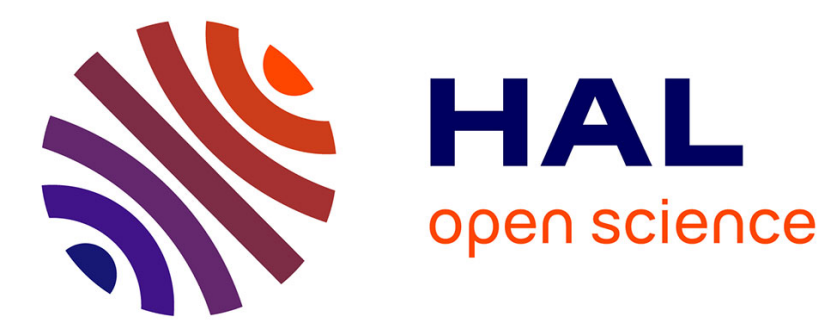

\title{
Special section Industry 4.0: Challenges for the future in manufacturing
}

Hervé Panetto, Georg Weichhart, Roberto Pinto

\section{To cite this version:}

Hervé Panetto, Georg Weichhart, Roberto Pinto. Special section Industry 4.0: Challenges for the future in manufacturing. Annual Reviews in Control, 2019, 47, pp.198-199. 10.1016/j.arcontrol.2019.04.002 . hal-02090830

\section{HAL Id: hal-02090830 https://hal.science/hal-02090830}

Submitted on 5 Apr 2019

HAL is a multi-disciplinary open access archive for the deposit and dissemination of scientific research documents, whether they are published or not. The documents may come from teaching and research institutions in France or abroad, or from public or private research centers.
L'archive ouverte pluridisciplinaire HAL, est destinée au dépôt et à la diffusion de documents scientifiques de niveau recherche, publiés ou non, émanant des établissements d'enseignement et de recherche français ou étrangers, des laboratoires publics ou privés. 
Special section on Industry 4.0: challenges for the future in manufacturing

Hervé Panetto, Université de Lorraine, CNRS, CRAN, France, Georg Weichhart, PROFACTOR, Johannes Kepler University, Austria, Roberto Pinto, University of Bergamo, Italy

\section{Introduction}

The sensing enterprise is a digital business innovation concept making Cyber-Physical Systems, service-oriented architectures and advanced human-computer interactions converge, supporting a more agile, flexible, and proactive management of unexpected events in today's global value networks. In essence, it concerns the adoption of future Internet technologies in virtual enterprises. Translating this concept to a general approach to smart systems (smart manufacturing, smart cities, smart logistics, etc.), requires new capabilities by next-generation information systems to perform sensing, modelling, and interpretation of "any" signal from the real world, thus providing the systems with higher flexibility and possibilities for reconfiguration (Panetto et al. 2016). Intuitively, a sensing system requires resources and machineries to be constantly monitored, configured, and easily controlled by human operators. All these functions, and much more indeed, are now implemented by the so-called (Industrial) Internet of Things or Cyber-Physical Systems. With the advent of the new cyber-physical system design paradigm, the number and diversity of systems that need to work together in the future enterprises have significantly increased (Weichhart et al. 2016). This trend highlights the need to shift from the classic central control of systems, towards systems interoperability as a capability to control, sense, and perceive distributed and heterogeneous systems and their environments, as well as to purposefully and socially act upon their perceptions. Such a shift could have important consequences on the future architecture design of the control of these systems. The emergence of cloud-based technologies will also have a significant impact on the design and implementation of cyber-physical systems; using such novel technologies, collaborative engineering practises will increase globally, thus enabling a new generation of small-scale industrial organizations to function in an informationcentric manner and enabling industry 4.0 transformations (Cimini, et al, 2017). The potential of such technologies in fostering a leaner and more agile approach towards engineering is very high. Engineers and engineering organizations no longer have to be restricted to the availability of advanced processing capabilities, as they can adopt a 'pay as you go' approach, which will enable them to access and use software resources for engineering activities from any remote location in the world.

\section{Outline of the section}

The 16th IFAC Symposium on Information Control Problems in Manufacturing (IFAC INCOM 2018), held in Bergamo, Italy in June 2018 aimed at providing a context facilitating the knowledge exchange, the review and discussion of theoretical advances, research results, and industrial experiences, among scientists, researchers, decision makers, practitioners and students. The general theme of INCOM 2018 was "Information Control for Responsive Manufacturing Systems". The true capability to achieve such a responsiveness depends substantially on the progress in advanced technologies and innovative methodologies at all levels of design, manufacturing and control of the industrial infrastructure (INCOM 2018). 
Extended versions of a selection of papers presented at the sessions organised in the frame of the IFAC Coordinating Committee 5 (CC5) in "Manufacturing and Logistics systems" have been peer reviewed, and six of them have been accepted for this special section of the journal dealing with "Industry 4.0: challenges for the future in manufacturing".

The first paper of this section, authored by Hervé Panetto, chair of the IFAC Coordination Committee CC5, and by the chairs of the four involved Technical Committees (TC), respectively Benoit Iung, Dimitry Ivanov, Georg Weichhart, and Xiaofan Wang is titled "Challenges for the Cyber-Physical Manufacturing Enterprises of the Future". This paper summarizes a vision of the challenges facing the so-called "Industry of the Future" as studied by the research community of the IFAC Coordinating Committee 5. Each TC brings its own vision and puts forward trends and issues important and relevant for future research. The analysis is performed on the enterprise-level topics with an interface to other relevant systems (e.g., supply chains). The vision developed, supports the identification of new scientific control directions such as Industry 4.0 technology-enabled new production strategies that require highly customised supply network control, the creation of resilient enterprise approaches to cope with risks, developments in management decision-support systems for the design, scheduling and control of resilient and digital manufacturing networks, and collaborative control. Due to the increasing number of elements and systems, complex and heterogeneous enterprise systems need to be considered (e.g., for decision-making). These systems are heterogeneous and built by different stakeholders. To make use of these, an environment is needed that allows the integration of the systems forming a System-of-Systems (SoS). The vision of the overall contribution from the research community in manufacturing and logistics systems over the next few years is to bring together researchers and practitioners presenting and discussing topics in modern manufacturing modelling, management and control in the emerging field of Industry 4.0-based resilient and innovative production SoS and supply networks.

The second paper, authored by Andrew Kusiak, is titled "Fundamentals of smart manufacturing: A multi-thread perspective". This paper outlines key characteristics of smart manufacturing, data-driven, networked, connected, resource sharing, resilient, and sustainable. Manufacturing resiliency and sustainability have received limited attention in the literature and they are the focus of this paper. Both are related and offer challenges that may become differentiators of smart manufacturing. Resiliency provides businesses with defences against natural and human caused adversities. The list of attributes provided in the paper is intended for comprehensive assessment of manufacturing resiliency. Solutions are needed to make businesses and more precisely the control of the systems more resilient and sustainable. Research on business models equating sustainability with an industrial activity is suggested. A scheme for labelling environmental friendliness of materials makes as a token contribution to sustainability.

The third paper authored by Francesco Longo, Letizia Nicoletti, and Antonio Padovano is titled "Ubiquitous knowledge empowers the Smart Factory: The impacts of a Service-oriented Digital Twin on enterprises' performance”. While the Industry 4.0 is idolizing the potential of an artificial intelligence embedded into "things", the role of the human component is neglected, even if indispensable in different manufacturing activities. This paper proposes an Industrial Internet pyramid as emergent human-centric manufacturing paradigm within Industry 4.0 in which central is the role of a Ubiquitous Knowledge about the manufacturing system intuitively accessed and used by the manufacturing employees. Second, the prototype of a Service-oriented Digital Twin, which leverage on a flexible ontology-oriented knowledge structure and on augmented reality combined to a vocal interaction system for an intuitive knowledge retrieval and fruition, has been designed and developed to deliver this manufacturing 
knowledge. Two test-beds, complimentary for the problems in practice (the former on the maintenance-production interface in a large enterprise, the latter majorly focused in production and setups in a small and medium enterprise), show the significant benefits in terms of time, costs and process quality, thus validating the approach proposed. This paper shows that a human-centric and knowledge-driven approach can drive the performance and the control of Industry 4.0 systems and lead a Smart Factory towards its full potential.

The fourth paper, authored by Kleanthis Thramboulidis, Danai C. Vachtsevanou, and Ioanna Kontou discusses "CPuS-IoT : A Cyber-Physical Microservice and IoT-based Framework for Manufacturing Assembly Systems”. While Assembly Systems (AS) often have a built-in flexibility in workshop like layouts, the control system needs to be as flexible as the AS. Flexibility increases the complexity, which in turn translates to computational power needs for the control system. The authors have created an architecture where sensor data is transferred to edge and fog computing, compressed, processed and only then send to cloud services. To have a scalable system, micro-services are used to contain the necessary algorithms. The architecture is used for the planning of assembly processes. To automate the planning of assembly processes several data-processing services are needed. However, this automated planning supports the required flexibility needed in today's cyber physical systems.

This fifth paper, authored by Christos Emmanouilidis, Petros Pistofidis, Luka Bertoncelj, Vassilis Katsouros, Apostolos Fournaris, Christos Koulamas, and Cristobal Ruiz-Carcel addresses the efficient integration of human and non-human actors in sociotechnical systems and Cyber-Physical environments under the title "Enabling the Human in the Loop: Linked Data and Knowledge in Industrial Cyber-Physical Systems”. Indeed, incorporating human skills and tangible knowledge requires approaches and technological solutions that facilitate the engagement of personnel within technical systems. The potential added value can be amplified, through solutions that allow a seamless data, information, knowledge, and decision flows. This paper presents examples of technology enablers for placing the human in the loop at selected application cases, thereby introducing a novel viewpoint for enabling human in the loop engagement linked to cognitive capabilities, and highlighting the role of context information management in industrial systems.

The last paper, authored by Michele Dassisti, Antonio Giovannini, Pasquale Merla, Michela Chimienti, and Hervé Panetto, is titled "An approach to support Industry 4.0 adoption in SMEs using a core-metamodel". Despite the recent growing interest in the "factory smartness", still there are only few small and medium enterprises (SMEs) that adopt effective Industry 4.0 (I4.0) solutions. The main reasons can be related to the lack of formalized processes, lack of ICT knowledge as well as low-cost commercial systems. To cope with these issues, this paper focuses on the development and the application of an approach to provide SMEs with a multi-purpose, modular, knowledge-based system: the main aim is to provide a modular and extensible system that can be incrementally implemented without requiring huge initial investments. This system is based on a core design-knowledge meta-model. From this core meta-model, multi-purposes modules can be built: in this paper, we present modules for the traceability support, the AR-powered assembly support, the machine-to-machine control and the data analysis support.

The Editors of this special section of Annual Reviews in Control would like to express their gratitude to the authors for their excellent contributions. We are also very grateful to all reviewers who have dedicated efforts in reviewing these papers, and for their valuable comments and suggestions that significantly improved the overall quality of the contributions. 
We hope that this special section will serve as a good reference for researchers, scientists, engineers, and academicians in the field of the industry of the future.

\section{References}

INCOM 2018. Website of the 16th IFAC Symposium on Information Control Problems in Manufacturing,11-13 June 2018, Bergamo Italy. http://incom2018.org/

Hervé Panetto, Milan Zdravković, Ricardo Jardim-Goncalves, David Romero, J. Cecil, István Mezgár (2016). New Perspectives for the Future Interoperable Enterprise Systems. In Computers in Industry, Elsevier, 2016, Special Issue: "Future Perspectives on Next Generation Enterprise Information Systems: Emerging Domains and Application Environments”, 79, pp. 47-63. 〈10.1016/j.compind.2015.08.001〉

Georg Weichhart, Arturo Molina, David Chen, Lawrence E. Whitman, François Vernadat (2016). Challenges and current developments for Sensing, Smart and Sustainable Enterprise Systems. Computers in Industry, Elsevier, 2016, Special Issue: "Future Perspectives on Next Generation Enterprise Information Systems: Emerging Domains and Application Environments”, 79, pp. 34-46. 〈10.1016/j.compind.2015.07.002〉

Chiara Cimini, Roberto Pinto, Giuditta Pezzotta, Paolo Gaiardelli P. (2017) The Transition Towards Industry 4.0: Business Opportunities and Expected Impacts for Suppliers and Manufacturers. In: Lödding H., Riedel R., Thoben KD., von Cieminski G., Kiritsis D. (eds) Advances in Production Management Systems. The Path to Intelligent, Collaborative and Sustainable Manufacturing. APMS 2017. IFIP Advances in Information and Communication Technology, vol 513. Springer. 〈10.1007/978-3-319-66923-6_14〉 